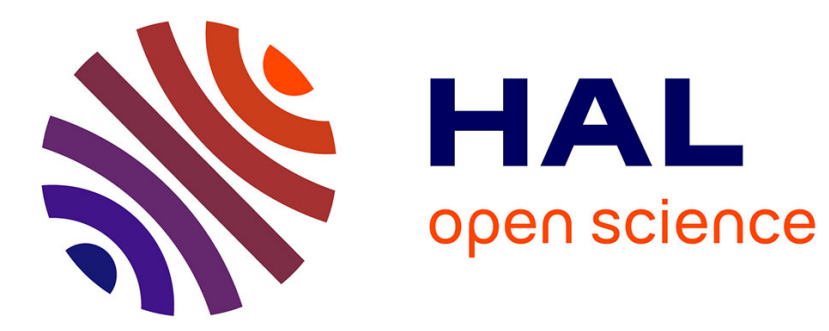

\title{
Cell autonomous commitment to an endodermal fate and behaviour by activation of Nodal signalling
}

\author{
Nicolas B. David, Frédéric M. Rosa
}

\section{To cite this version:}

Nicolas B. David, Frédéric M. Rosa. Cell autonomous commitment to an endodermal fate and behaviour by activation of Nodal signalling. Development (Cambridge, England), 2001. hal-02362156

\section{HAL Id: hal-02362156 \\ https://hal.science/hal-02362156}

Submitted on 13 Nov 2019

HAL is a multi-disciplinary open access archive for the deposit and dissemination of scientific research documents, whether they are published or not. The documents may come from teaching and research institutions in France or abroad, or from public or private research centers.
L'archive ouverte pluridisciplinaire HAL, est destinée au dépôt et à la diffusion de documents scientifiques de niveau recherche, publiés ou non, émanant des établissements d'enseignement et de recherche français ou étrangers, des laboratoires publics ou privés. 


\title{
Cell autonomous commitment to an endodermal fate and behaviour by
}

\section{activation of Nodal signalling}

\author{
Nicolas B. David and Frédéric M. Rosa* \\ Groupe Danio, U 368 INSERM, Ecole Normale Supérieure, 46, rue d' Ulm, F-75230 Paris Cedex 05, France \\ *Author for correspondence (e-mail: rosa@wotan.ens.fr) \\ Accepted 20 July 2001
}

\section{SUMMARY}

In vertebrates the endoderm germ layer gives rise to most tissues of the digestive tract and controls head and heart morphogenesis. The induction of endoderm development relies on extracellular signals related to Nodals and propagated intracellularly by TGF $\beta$ type I receptors ALK4/Taram-A. It is unclear, however, whether Nodal/ALK4/Taram-A signalling is involved only in the specification of endodermal precursors or plays a more comprehensive role in the activation of the endodermal program leading to the irreversible commitment of cells to the endodermal fate. Using cell transplantation experiments in zebrafish, we show that marginal cells become committed to endoderm at the onset of gastrulation and that commitment to endoderm can be reached by intracellular activation of the Nodal pathway induced by expression of an activated form of the taram-A receptor, Tar*. In a manner similar to endoderm progenitors, Tar*activated blastomeres translocate from their initial site of implantation in the blastoderm to reach the surface of their migration substratum, the yolk syncitial layer, where

\section{INTRODUCTION}

The endoderm germ layer plays essential functions during vertebrate embryogenesis, first because it populates the digestive and respiratory tracts but also because it interacts with the other germ layers to control the proper morphogenesis of numerous structures and organs including the head and the heart (Wells and Melton, 1999). In zebrafish, endoderm progenitor cells originate, shortly before the onset of gastrulation, from the outside layer of the blastoderm within four cells diameters from the margin of the embryo. At the onset of gastrulation, they accomplish an inward movement to reach their future migration substratum, the yolk syncitial layer (YSL) a large spherical extraembryonic compartment underlying the blastoderm (Warga and Nusslein-Volhard, 1999). During gastrulation, endoderm progenitors migrate on the YSL surface to form the endoderm germ layer and remain on this substratum until they initiate their local differentiation (Warga and Nusslein-Volhard, 1999).

The mechanisms involved in the control of endodermal fate and behaviour are only partially understood. Endoderm they join endogenous endodermal derivatives during gastrulation and differentiate according to their anteroposterior position. We demonstrate that Nodal/Tar*. induced commitment does not rely on a secondary signal released by Tar*-expressing cells or a signal released by endogenous endoderm since Tar*-expressing wild-type cells can restore endoderm derivatives when transplanted into the endoderm-deficient mutant casanova. Likewise, the YSL does not appear essential for the maintenance of endodermal identity during gastrulation once the Nodal pathway has been activated. Thus, our results demonstrate that the activation of Nodal signalling is sufficient to commit cells both to an endodermal fate and behaviour. Wild-type endoderm implantation into casanova embryos rescues, in a non-autonomous fashion, the defective fusion of the two heart primordia in the midline, highlighting the importance of endoderm for normal heart morphogenesis.

Key words: Endoderm, Nodal, Fate determination, Gastrulation, casanova, Heart, Zebrafish specification has been mainly analysed in Xenopus in which a two-step model was proposed (Dale, 1999; Yasuo and Lemaire, 1999). Maternal factors located in vegetal blastomeres, such as VegT, would activate the zygotic expression of both early endodermal transcription factor and TGF $\beta$-related molecules such as Nodal related 1 or 2 and Derrière. In a second step, these zygotically expressed TGF $\beta$-related genes would relay VegT in activating endodermal genes. In zebrafish, genetic studies have led to a model of endoderm specification that shares several zygotic steps with the Xenopus model (Alexander and Stainier, 1999). Embryos mutant for Nodalrelated genes cyclops (cyc) and squint (sqt), or lacking both maternal and zygotic Nodal cofactor OEP (Gritsman et al., 2000), form essentially no endoderm or mesoderm (Feldman et al., 1998; Rebagliati et al., 1998). Conversely, activation of the Nodal /activin pathway by Tar*, an activated version of the type I TGF $\beta$-related receptor, Taram-A (Tar) (Renucci et al., 1996) which is normally expressed in a region encompassing endodermal progenitors, drives blastomeres to an endodermal fate and rescues endoderm formation in oep mutants (BallyCuif et al., 2000; Peyrieras et al., 1998). This suggests that the 
activation of the Nodal/Tar pathway directly induces endoderm formation, but it is not clear whether other signals are also required.

Transcription factors like the Mix-type homeobox protein Bon (bonnie and clyde), and Fau/Gata5 (faust) have been implicated downstream of Nodal-related signals. Both are induced by Nodal-related molecules, and the number of endodermal cells is reduced in the bon and fau mutants (Alexander and Stainier, 1999; Kikuchi et al., 2000; Reiter et al., 2001). Endoderm formation also requires Cas (casanova), a Sox-related factor (Alexander et al., 1999; Dickmeis et al., 2001; Kikuchi et al., 2001). Epistatic studies have shown that cas functions downstream of cyc, sqt, oep, bon and fau. In particular, cas embryos are not able to respond to Nodal signalling, whether activated by Nodal-related ligands or by expression of Tar*. Finally, a model for endoderm specification has been assembled, based on these results: Nodal-related proteins induce the expression of transcription factors like Bon, Fau/Gata5 that allow the expression of cas. In turn, Cas initiates, in an instructive manner, the expression of endodermal genes such as sox 17 (Reiter et al., 2001) and downregulates mesodermal genes (Dickmeis et al., 2001; Kikuchi et al., 2001; and our own unpublished data).

Although the molecular mechanisms implicated in endoderm specification start to be unravelled, very little is known about the signals and molecules required for the determination/commitment of a cell to an endodermal fate. Determination/commitment of a cell or a group of cells is defined by the acquisition of a fate that cannot be modified by transplantation into a new embryonic environment (Spemann, 1938). Cell transplantation experiments in Xenopus have shown that commitment to the endoderm germ layer occurs at the early gastrula stage (Heasman et al., 1984; Wylie et al., 1987). However, this point has not been addressed in zebrafish, and the signals controlling this commitment remain unidentified in either model. In particular, it is not clear whether signals able to specify endoderm, such as Nodals, also participate in the determination process.

Endoderm cells undergo an extensive migration period which is critical for the establishment of the endoderm germ layer and probably its later patterning, since cells can receive extrinsic patterning cues depending on their environment (Grapin-Botton and Melton, 2000; Joubin and Stern, 1999). It is thus important to understand what controls the induction of the behaviour of endoderm cells during gastrulation, what drives their segregation from the other cells of the embryo and whether this segregation requires that endoderm cells be present at the margin of the embryo. The capacity to specifically orient the early blastomeres into the endoderm pathway should help clarify this issue.

In this study, using cell transplantation experiments, we have first defined the timing for endoderm commitment and the properties of committed cells. We show that endoderm-fated cells reach a committed state at the onset of gastrulation and that animal pole blastula cells, normally fated to neuroectoderm (Kimmel et al., 1990), when transplanted to the margin can adopt an endodermal fate, indicating that marginal signals, potentially Nodals, are sufficient to induce the endodermal program. We have tested the implication of Nodal signals in these processes and found that cells in which the Nodal pathway is activated by Tar* (which for the sake of clarity, will simply be called 'Tar*-activated cells' or 'activated cells' throughout this article) adopt an endodermal identity even when grafted to the animal pole. Furthermore both endoderm-committed cells and activated cells translocate to the YSL surface when transplanted into any position of the blastoderm, demonstrating that once committed, endoderm cells can segregate from other cell types and that this behaviour can be induced by activation of Nodal signalling. Then Nodal/Tar*-activated cells systematically join endogenous endodermal derivatives during gastrulation and differentiate according to their anteroposterior position. Thus, once activation of Nodal signalling is initiated, cells are committed both to an endodermal fate and behaviour. This commitment does not rely on a community effect between Tar*-activated cells and endoderm identity does not require the presence of the YSL during gastrulation. It also appears independent of endogenous endoderm since wild-type cells expressing Tar* can restore endodermal derivatives in a mutant deprived of endoderm such as cas. Taking advantage of this last result, we have analysed the origin of the cardia bifida in this mutant, a heart condition resulting from the absence of fusion of the primordial heart tubes, often attributed to an absent or defective endoderm (Narita et al., 1997; Peyrieras et al., 1998). Cardia bifida can be rescued by introduction of wild-type endoderm into cas embryos, thus demonstrating that cas activity is not required within heart precursors and strengthening the notion that endoderm is a key element controlling normal heart morphogenesis.

\section{MATERIALS AND METHODS}

\section{Embryos}

Embryos were obtained from wild-type $(\mathrm{AB})$ or heterozygous cas $^{\mathrm{ta} 56}$ (Chen et al., 1996) adults and raised according to previously published procedures (Kimmel et al., 1995).

\section{Grafting experiments}

Donor embryos at the 2/4-cell stage were injected in one blastomere with $80 \mathrm{pg}$ of $G F P+120 \mathrm{pg}$ of nls-lacZ mRNAs used as lineage tracers, either alone or combined with $1.2 \mathrm{pg}$ of $\operatorname{Tar}^{*} \mathrm{mRNA}$. At the sphere stage, 5-20 donor (green) cells were grafted to the margin or to the animal pole of host embryos (Ho and Kimmel, 1993). Embryos were then cultured in embryo medium (Westerfield, 1995) with 10 $\mathrm{U} / \mathrm{ml}$ penicillin and $10 \mu \mathrm{g} / \mathrm{ml}$ streptomycin. The dorsoventral position of grafts was evaluated by the shield stage. For single cell transplantations, donor embryos, once injected, were cultured in calcium-free Ringer medium. At the sphere stage, cells were mechanically dissociated and isolated cells were transplanted.

In determination experiments, embryos were injected with the activatable dye DMNB-FD, which was activated at the $30-40 \%$ epiboly stage (see Bally-Cuif et al., 2000; Serbedzija et al., 1998) in a few cells and used as a source of cells for transplantation. This procedure allowed us to test the evolution in time of the properties of cells originating from the same region of the fate map.

\section{Animal caps culture}

Once cells had been grafted to the animal pole, embryos were placed in MMR medium (Kimelman and Maas, 1992). Animal caps were dissected with forceps and cultured in L15 (65\%) (Gibco), embryo medium (20\%), BSA $(1 \mathrm{mg} / \mathrm{ml})$, Hepes $(\mathrm{pH} 7.510 \mathrm{mM})$, penicillin, streptomycin and $\mathrm{H}_{2} \mathrm{O}$. They were fixed when control embryos had reached $90 \%$ epiboly. 


\section{Phenotypic analyses}

In situ hybridisation and immunohistochemistry were done following standard protocols (Hauptmann and Gerster, 1994). Probes used were: foxA2 (Strahle et al., 1993), fkd7 (Odenthal and Nusslein-Volhard, 1998), gata5 (Rodaway et al., 1999), her5 (Muller et al., 1996), insulin (Argenton et al., 1999), $n k x 2.3$ (Lee et al., 1996), $n k x 2.5$ (Lee et al., 1996), sox17 (Alexander and Stainier, 1999). For the immunodetection of $\beta$-galactosidase, rabbit polyclonal antibodies (Cappel) were used at 1:1000 dilution. Sectioning of whole-mount stained embryos was performed with JB4 embedding resin (Polysciences).

\section{RESULTS}

\section{Endodermal progenitors become determined around the onset of gastrulation}

To define the timing for endoderm commitment, lineagelabelled marginalmost blastomeres or animal blastomeres, as the control, were transplanted (Fig. 1A) from late blastula-early gastrula wild-type donors to the animal pole region of host sibling embryos. Consistent with the fate map, transplanted animal pole cells contributed to neuroectodermal but never to endodermal tissues (Fig. 1B, 100\%, $n=11$ embryos). In contrast, marginalmost blastomeres transplanted from late blastulae (30-40\% epiboly), contributed mostly to neuroectodermal tissue but in a fraction of the embryos, a small proportion of transplanted cells contributed to endodermal derivatives (data not shown). This contribution to endodermal derivatives increased with the age of the donor embryos since marginal cells transplanted from embryos initiating gastrulation (50\% epiboly-germ ring stage), populated mostly the anterior gut tube region (pharyngeal epithelium; Fig. 1C, $83 \%, n=23$ embryos) and expressed the late endoderm derivative marker $f k d 7$ (Fig. 1D). Thus, at the onset of gastrulation, determination of marginal cells to an endodermal fate is largely under way. The properties of these committed cells was further investigated, and in the text they will be referred to as ECB (endoderm committed blastomeres).

\section{Cells grafted to the margin acquire an endodermal fate}

Marginal cells could develop an endodermal fate either because they have inherited determinants or because they receive inductive signals from their environment. To discriminate between these two possibilities, we tested whether animal pole cells, normally fated to the neuroectoderm, would be able to populate endodermal territories when grafted to an endoderm fated region. If determinants are essential, animal pole cells should not be able to populate endoderm-derived territories. Animal pole cells expressing GFP as a lineage tracer were grafted at the blastula stage (high-sphere stage) to the margin or to the animal pole of host sibling embryos (Fig. 2A) and allowed to develop. Whereas cells grafted to the animal pole developed into neuroectodermal structures (Fig. 2G,H; Table $1)$, progeny of cells grafted at the margin populated predominantly endoderm and mesoderm (Fig. 2B-D; Table1), consistent with the fate map and could differentiate into endodermal structures like the gut and the pharyngeal epithelium (data not shown). In these experiments we found it crucial for grafted cells to remain near the margin to develop an endodermal fate. Thus animal pole cells can assume an endodermal fate when transplanted to the margin, showing that region-specific determinants are not absolutely required but that environmental cues, potentially Nodals, are sufficient to trigger the endodermal program.

\section{Tar*-expressing cells are committed to an endodermal fate}

To test the influence of Tar*/Nodal signalling on the state of determination of early blastomeres, a few GFP-expressing Tar*-activated cells were transplanted from late blastulae (sphere stage) to the margin or to the animal pole of untreated embryos. In $24 \mathrm{hpf}$ (hours postfertilisation) embryos, in contrast to GFP-expressing cells, almost all Tar*-activated cells were located onto the YSL, a position corresponding to that of the endogenous endoderm. Moreover, whereas Tar*activated cells grafted to the animal pole developed into pharyngeal endoderm and into hatching gland, a mesendodermal prechordal plate derivative (Fig. 2I; Table 1), progeny of cells grafted in marginal positions could be found at any position of the anteroposterior (AP) axis and took part in all endodermal derivatives: pharynx, intestine (Fig. 2E,F) and the mesendodermal derivatives, hatching gland and Kuppfer's vesicle (Table 1). Furthermore, grafted Tar*activated cells expressed endodermal markers in register with their AP position: cells below the head expressed the
Fig. 1. Endoderm commitment occurs at the onset of gastrulation. (A) Diagram of the experimental procedure. DMNB-caged fluorescein was injected at the 1-cell stage. At the late blastula stage, fluorescein was uncaged in a few cells at the animal pole or at the very margin of the donor embryo. At $50 \%$ epiboly-germ ring, labelled cells were transplanted to the animal pole of a late blastula (dome) host. (B,C) Anterior to the left, dorsal to the top. (B) Cells transplanted from the animal pole of the donor populated the eye and the forebrain and differentiated accordingly. (C) In contrast, marginalmost cells taken just before or at the onset of gastrulation colonised the endoderm-derived territories and expressed the endodermal marker $f k d 7$ (section in D). Some of these cells also populated the hatching gland (not shown). The white dots indicate the limit of the YSL. e, eye; no, notochord; nt, neural tube; AP, Animal Pole; M, Margin.

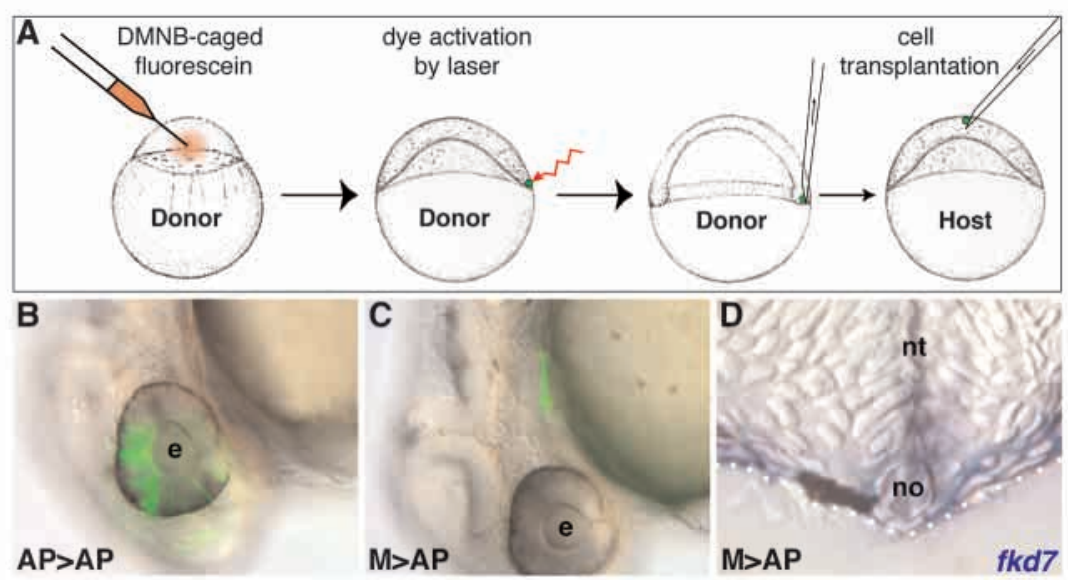




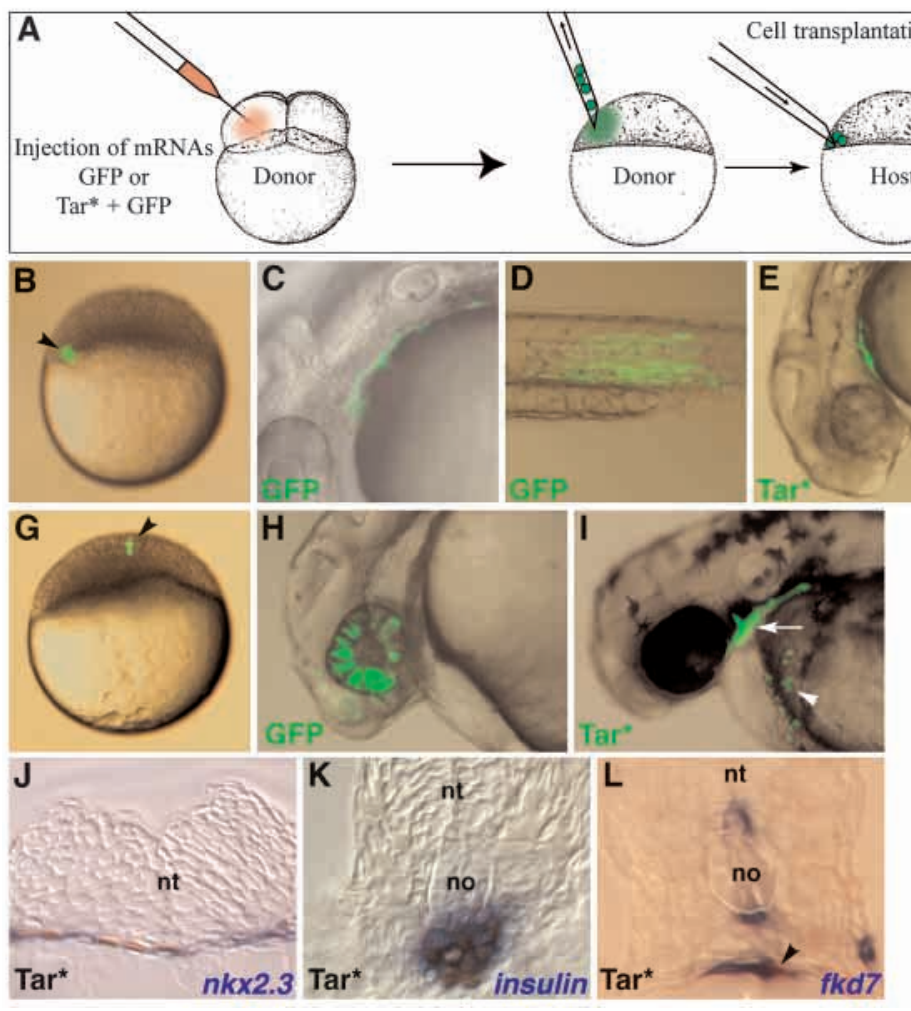

pharyngeal endoderm marker $n k x 2.3$ (Fig. 2J) (Lee et al., 1996) whereas cells in the trunk expressed the gut marker $f k d 7$ (Fig. 2L) (Odenthal and Nusslein-Volhard, 1998) and cells in the pancreas expressed insulin (Fig. 2K) (Argenton et al., 1999). In addition, the graft of Tar*-activated cells did not induce ectopic sites of expression of these regional marker genes. Thus, endodermal derivatives formed by grafted cells differentiate in register with their AP position. Besides, AP fates adopted by Tar*-activated grafted cells were in agreement with the fate map of endodermal derivatives established at the beginning of gastrulation (Warga and Nusslein-Volhard, 1999).
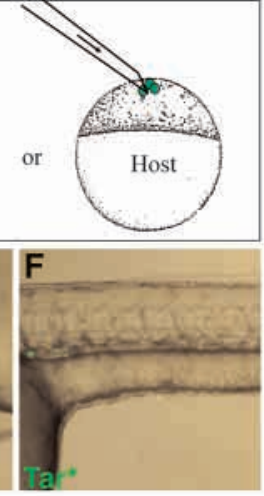

mesodermal derivatives (D) (see Table

1). By contrast, activated (Tar* + GFP) cells almost exclusively become endodermal derivatives, such as pharynx $(\mathrm{E})$ and gut $(\mathrm{F})$, and mesendodermal hatching gland. (H) GFP-expressing cells transplanted to the animal pole populate the eye and the forebrain, whereas (I) Tar*-expressing cells contribute progeny only to the endodermal pharynx (white arrow) and to mesendodermal derivatives (hatching gland, white arrowhead). (J-L) Transverse sections showing the expression of the endodermal markers $n k x 2.3$ $(\mathrm{J})$, insulin $(\mathrm{K})$ and $f k d 7$ (L, arrowhead) by grafted Tar*-expressing cells. In all the experiments, nls-lac $Z$, which encodes nuclear $\beta$ galactosidase was coexpressed with GFP as a lineage tracer for immunohistochemistry (brown nuclei) to identify grafted cells. Dorsal to the top. no, notochord; nt, nerve tube.

Cells grafted in dorsal positions in host gastrulae mainly gave rise to anterior derivatives such as the epithelium of the pharynx, whereas transplantations into more ventral positions resulted in more posterior fates, such as the gut (Table 1). We conclude that activation of Nodal/Tar signalling is sufficient to induce the stable commitment of early zebrafish blastomeres to an endodermal fate. Furthermore, activated cells are submitted to proper AP patterning.

Commitment, here assessed by transplantation to the animal pole, could however result from a late event, like the recruitment of transplanted cells by the endogenous endoderm

Table 1. Results of cell transplantations

\begin{tabular}{|c|c|c|c|c|c|c|c|c|c|c|c|c|c|c|c|}
\hline & \multicolumn{2}{|c|}{ Transplantation } & & \multirow[t]{2}{*}{ 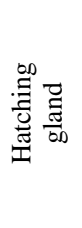 } & \multirow[t]{2}{*}{ 胥 } & \multirow[t]{2}{*}{ 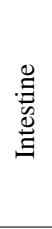 } & \multirow[t]{2}{*}{ 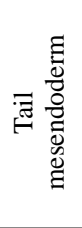 } & \multirow[t]{2}{*}{$\begin{array}{l}\bar{D} \\
\overline{0} \\
\frac{d}{0} \\
0 \\
0 \\
z\end{array}$} & \multirow[t]{2}{*}{$\begin{array}{l}\mathscr{\mathscr { a }} \\
\stackrel{\mathscr{J}}{0} \\
\tilde{n}\end{array}$} & \multirow[t]{2}{*}{ 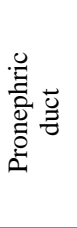 } & \multirow[t]{2}{*}{ 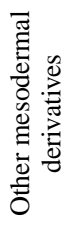 } & \multirow[t]{2}{*}{ 空 } & \multirow[t]{2}{*}{ 寻 } & \multirow[t]{2}{*}{ 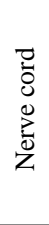 } & \multirow[t]{2}{*}{$\begin{array}{l}\tilde{a} \\
\bar{J} \\
\tilde{\Xi} \\
\tilde{\Xi}\end{array}$} \\
\hline & From & To & & & & & & & & & & & & & \\
\hline \multirow[t]{2}{*}{ GFP } & AP & M & $n=42$ & 10 & 36 & 14 & 0 & 19 & 86 & 17 & 48 & 0 & 2 & 31 & 5 \\
\hline & $\mathrm{M}$ & M & $n=40$ & 35 & 37 & 17 & 10 & 25 & 62 & 12 & 22 & 0 & 32 & 17 & 7 \\
\hline \multirow{2}{*}{ Tar*+GFP } & M & D M & $n=26$ & 23 & 96 & 65 & 15 & 15 & 0 & 0 & 0 & 0 & 15 & 4 & 0 \\
\hline & & Total & $n=102$ & 10 & 82 & 78 & 51 & 10 & 0 & 0 & 4 & 0 & 8 & 4 & 4 \\
\hline GFP & AP & $\mathrm{AP}$ & $n=19$ & 0 & 0 & 0 & 0 & 0 & 0 & 0 & 0 & 90 & 95 & 0 & 21 \\
\hline Tar*+GFP & $\mathrm{AP}$ & $\mathrm{AP}$ & $n=63$ & 29 & 97 & 21 & 3 & 0 & 0 & 0 & 0 & 0 & 10 & 0 & 11 \\
\hline
\end{tabular}

The phenotype of grafted cells was scored when host embryos had reached 24 to $30 \mathrm{hpf}$. The figures are the percentage of embryos with at least one cell participating in a given tissue. In each embryo, the number of cells incorporated into each type of derivative could not be precisely determined. However, the vast majority of Tar*-activated cells always adopted an endodermal or hatching gland fate, whereas only a few could be found in other derivatives. AP, animal pole; M, margin; V, ventral; L, lateral; D, dorsal. 
Fig. 3. Endoderm-committed blastomeres and activated cells adopt an endodermal identity at the onset of gastrulation. During gastrulation (60\% epiboly), endoderm-committed blastomeres (ECB) (A) or activated cells (C,D) transplanted to the animal pole (arrowheads) already express the endodermal genes sox 17 and her5. Control cells do not (B). (B-D) Insets show close-up views of the region containing grafted cells.
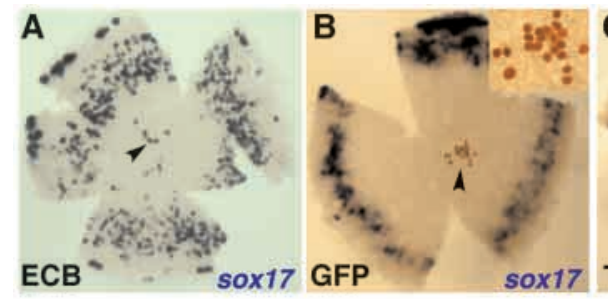

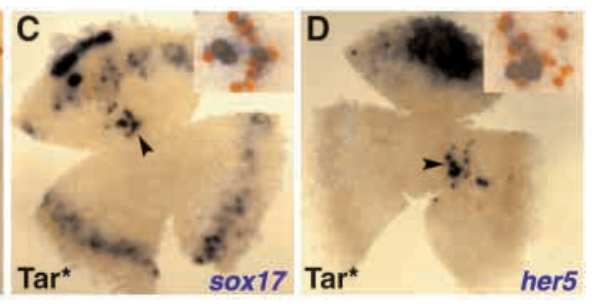

reaching the animal pole. To rule out this possibility, we checked that, when grafted to the animal pole, Tar*-activated cells as well as non-activated endoderm-committed blastomeres (ECB; Fig. 3A) adopted an endodermal identity before the migration of the endogenous endoderm to the animal pole. To this aim, we analysed the expression of the early endodermal markers sox 17 , her 5 , gata 5 and foxA 2 in the young gastrulae (60\% epiboly) harbouring grafted cells. ECB and Tar*-activated cells, but not control cells, grafted to the animal pole expressed these genes (sox $1776 \%, n=59$; her 5 $87 \%, n=8$; gata $543 \%, n=7$; foxA2 $100 \%, n=8$ for activated cells) (Fig. 3B-D; data not shown), thus demonstrating that endodermal specification was already initiated in Tar*activated cells by the beginning of gastrulation, and that the endodermal fate acquired by transplanted cells did not result from a late recruitment by the endogenous endoderm. Within individual embryos, some grafted cells did not express the tested gene, a situation that can be easily explained because endoderm markers do not label the mesendodermal hatching gland precursors, which are also induced by activation of Nodal/Tar signalling. Taken together, our results show that the endodermal program followed by Nodal/Tar*-activated cells is similar to that of endogenous endoderm but does not depend on additional signals released by the margin of the embryo.

\section{Autonomous commitment of cells activated by transient Nodal signalling}

In the experiments described above, we have always transplanted cells in groups. The acquisition of commitment to an endodermal fate observed upon intracellular activation of Nodal/Tar signalling might rely on contacts with neighbouring activated cells, in a so-called community effect (Gurdon, 1988). We used a single cell transplantation assay to test this hypothesis (Fig. 4A). Progeny of single Tar*-activated cells grafted to the animal pole or to the margin of host embryos, expressed sox 17 during gastrulation $(50 \%, n=16$; Fig. 4B), and became endodermal derivatives in $24 \mathrm{hpf}$ embryos $(100 \%, n=8$; Fig. 4C), thus demonstrating that a community effect was not required to reach commitment to an endodermal fate once Nodal signalling is activated. Taken together, the results of our transplantation experiments demonstrate that activation of Nodal signalling mediated by Tar is sufficient to induce the autonomous commitment of zebrafish early blastomeres to endoderm.

We then determined whether endoderm commitment could be induced by transient activation of Nodal signalling. Nodal signalling and endoderm formation require the transcription factor FAST1. Endoderm formation and endoderm marker expression during gastrulation can be blocked by overexpression of the dominant negative FAST-1 variant
FAST $^{\text {SID }}$ (Dickmeis et al., 2001; Muller et al., 2000). Consistent with this, expression of FASTSID by coinjection with Tar* at the 2-cell stage blocked the capacity of injected cells, when grafted to the animal pole of host embryos, to reach the YSL at the onset of gastrulation (not shown) and to achieve an endodermal fate (Fig. 4D,E; 0\%, $n=28$ ). We then delayed the expression of FAST ${ }^{\text {SID }}$ by injecting Tar* RNA at the 2-cell stage and FAST ${ }^{\text {SID }}$ RNA about an hour later at the 16-cell stage into Tar*-positive cells. Upon grafting, TAR* + FAST ${ }^{\text {SID }}$ expressing cells reached the YSL during gastrulation and adopted an endodermal fate (Fig. 4F,G; 61\%, n=23). Thus transient activation of Nodal signalling is sufficient to induce an autonomous commitment to endoderm.

\section{During gastrulation, activated and endoderm committed cells reach the surface of the YSL}

Fate determination concerns both cellular identity and location within the embryo. Previous results have shown that, even when grafted to the animal pole, activated cells or endoderm-

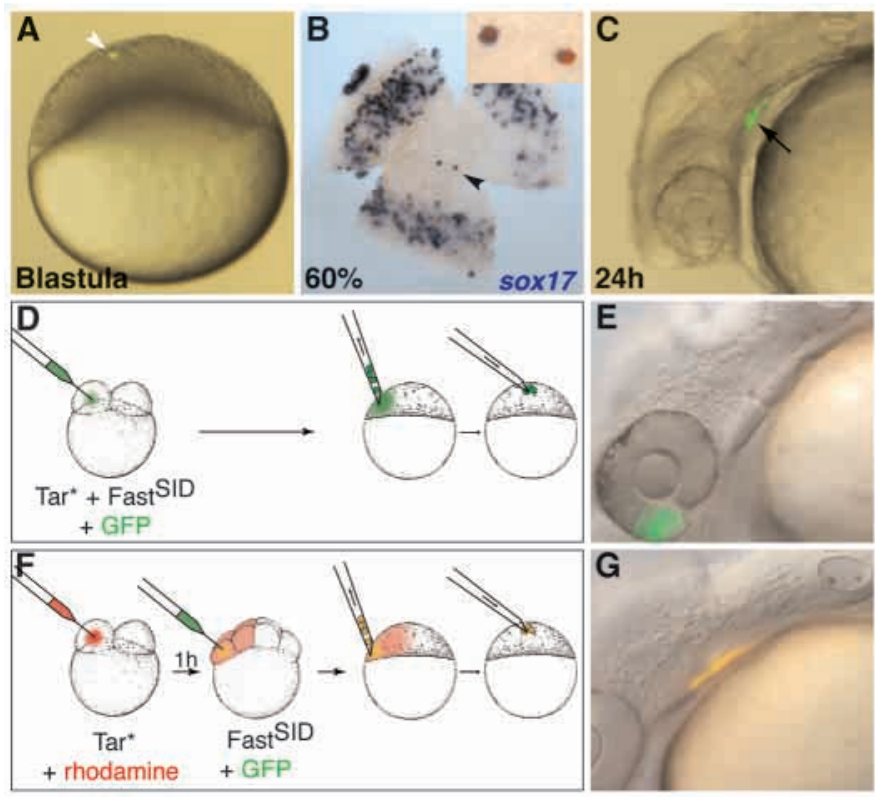

Fig. 4. Transient Nodal signalling autonomously commits cells to an endodermal fate. One single activated cell (white arrowhead) transplanted to the animal pole of a blastula host (A) expresses sox17 during gastrulation (arrowhead in B) and become endodermal derivatives at $24 \mathrm{hpf}$ (arrow in C). Co-injection of Tar* and Fast ${ }^{\mathrm{SID}}$ at the 2-cell stage (D) results in the cells that were transplanted to the animal pole being incorporated into the eye and the brain (E). By contrast, delayed injection of Fast ${ }^{\mathrm{SID}}(\mathrm{F})$ results in cells grafted to the animal pole adopting an endodermal fate $(\mathrm{G})$. 
Fig. 5. Endoderm-committed blastomeres and activated cells translocate directly to the YSL during gastrulation and develop an endodermal phenotype. Cells expressing GFP alone (A-D) or in combination with Tar* (E-H) or ECB (I-J) were transplanted to the animal pole of a host blastula. The group of grafted cells was monitored during development (lateral views). Until the beginning of gastrulation, grafted cells remain epiblastic (B,F,I), but during gastrulation, in contrast to control cells (C), Tar*-activated cells and ECB leave the epiblast to reach the surface of the YSL, without going through the margin $(\mathrm{G}, \mathrm{J})$.

$(\mathrm{D}, \mathrm{H})$ Morphology of a single control and an activated grafted cell during gastrulation (70\% epiboly). Note the filopodia characteristic of migrating endodermal cells in $\mathrm{H}$ (arrowheads).
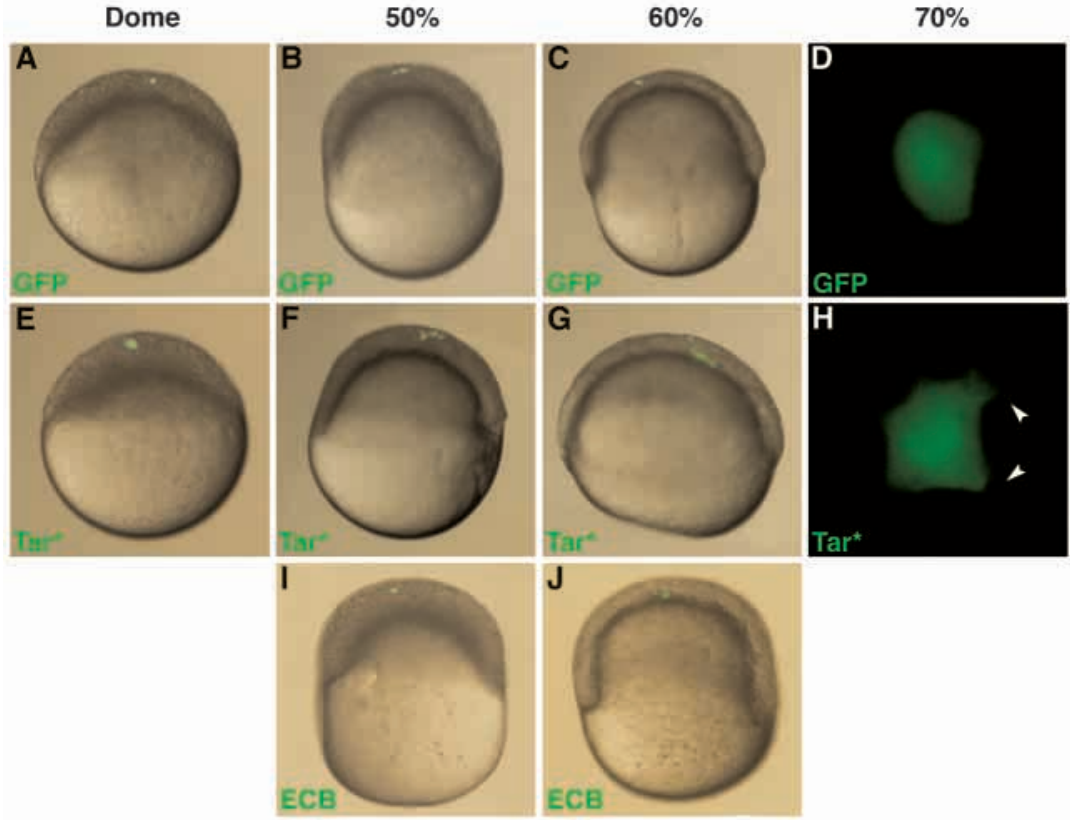

committed blastomeres (ECB) develop as endogenous endodermal derivatives in $24 \mathrm{hpf}$ embryos (Figs 1 and 2). This implies that, during development, activated cells/ECB leave the epiblast and reach the YSL. In vivo, this situation occurs at the margin of the embryo where cells involute to form the hypoblast. Do activated cells/ECB, when grafted to the animal pole, need to involute through the margin? To address this issue, we followed their behaviour by time-lapse analysis. Until the onset of gastrulation, both activated/ECB and control cells stayed epiblastic (Fig. 5A,B,E,F,I). During gastrulation, while control cells kept motionless (Fig. 5C), activated cells/ECB left their epiblastic location to reach the surface of the YSL, without coming to the margin of the blastoderm (Fig. $5 \mathrm{G}, \mathrm{J})$. There, in contrast to control cells (Fig. 5D), they acquired a flat morphology and developed filopodia (Fig. 4H), thereby adopting a location and a morphology typical of endodermal cells (Warga and Nusslein-Volhard, 1999). Thus activated cells/ECB are translocated to the hypoblast without the need to involute through the margin.

\section{The YSL is not necessary to maintain the endodermal identity of activated cells/ECB during gastrulation}

The YSL provides signals able to induce the formation of endoderm (Rodaway et al., 1999). In addition, during gastrulation, endogenous endoderm, as well as activated cells and ECB, come directly to and remain on the surface of the YSL. This suggests that the YSL could provide signals necessary to maintain endodermal identity, once Nodal signalling is activated. To test this hypothesis, small groups of activated cells or ECB were grafted to the animal pole of host blastulae and the animal caps of the host embryos were immediately dissected and grown until the end of gastrulation (90\% epiboly) (Fig. 6A,B). In both cases, ECB and activated cells remained within the cap and retained the expression of the endodermal marker sox 17 (respectively $80 \%, n=15$ caps and $100 \%, n=10$ caps; Fig. 6C,D). This result shows that grafted, activated cells or ECB retain their identity in the absence of the YSL, since they express sox17. Thus, once cells are committed to endoderm or once the Nodal/Tar* pathway is activated, the YSL does not seem absolutely essential for maintaining endodermal identity during gastrulation.

\section{Grafting wild-type activated cells can restore endoderm derivatives formation in casanova mutants}

Other regions of the embryo could provide signals important for cells to adopt an endodermal fate once Nodal/Tar signalling is activated. For instance, endoderm itself, which is in contact with transplanted cells during gastrulation, could be necessary. To test this idea and to address the genetic mechanisms responsible for the lack of endoderm and endoderm derivatives

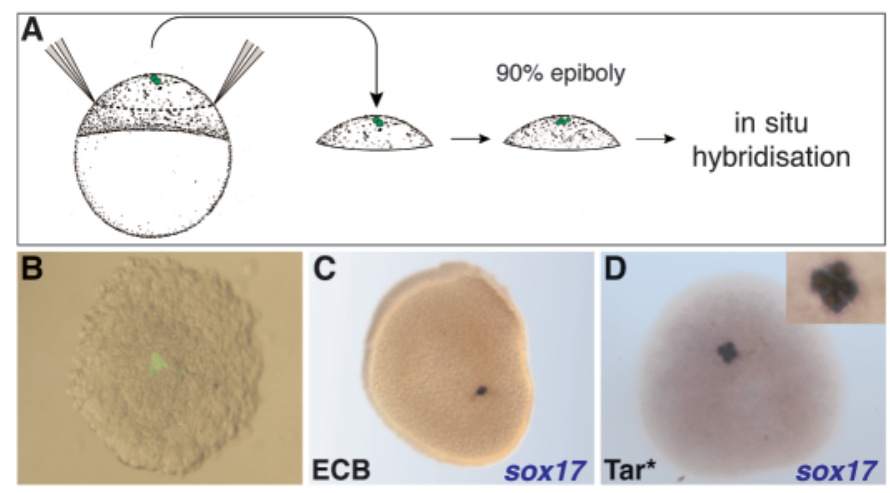

Fig. 6. The YSL is not required to maintain the endodermal identity of ECB and activated cells during gastrulation. (A) Diagram of the experimental procedure. At the blastula stage, activated cells (Tar* + GFP) or ECB were transplanted to the animal pole of host embryos. Animal caps containing the grafted cells (B) were immediately removed, cultured and fixed at the end of gastrulation (90\% epiboly). (C,D) sox 17 expression was revealed by in situ hybridisation (blue), and activated cells were identified by detection of nuclear $\beta$ galactosidase (brown, D). (D) Inset shows a close-up view of the region containing transplanted cells. 
in the cas mutant, activated cells were transplanted into cas mutant embryos. Grafting experiments have suggested that expression of the cas gene is required in a cell autonomous fashion inside endodermal progenitors to ensure proper expression of the transient endodermal marker foxA2 (Alexander et al., 1999). However, it is not clear from these experiments whether wild-type cells grafted into a cas mutant host can accomplish the full program of endoderm development and give rise to differentiated endodermal tissues. It is conceivable, in particular, that cas is required in cells other than endoderm or in the YSL. To investigate the requirement for endogenous endoderm in the control of the fate of activated cells, we tested whether wild-type Tar*-activated cells, when transplanted into cas mutant hosts could accomplish the whole endodermal program. By blastula stage, wild-type cells expressing Tar* were transplanted to the animal pole or to the margin of cas host embryos (Fig. 7A). During gastrulation, grafted activated cells expressed $\operatorname{sox} 17(33 \% n=9$; Fig. 7B) and her5 (data not shown). Moreover, in $24 \mathrm{hpf}$ embryos, they

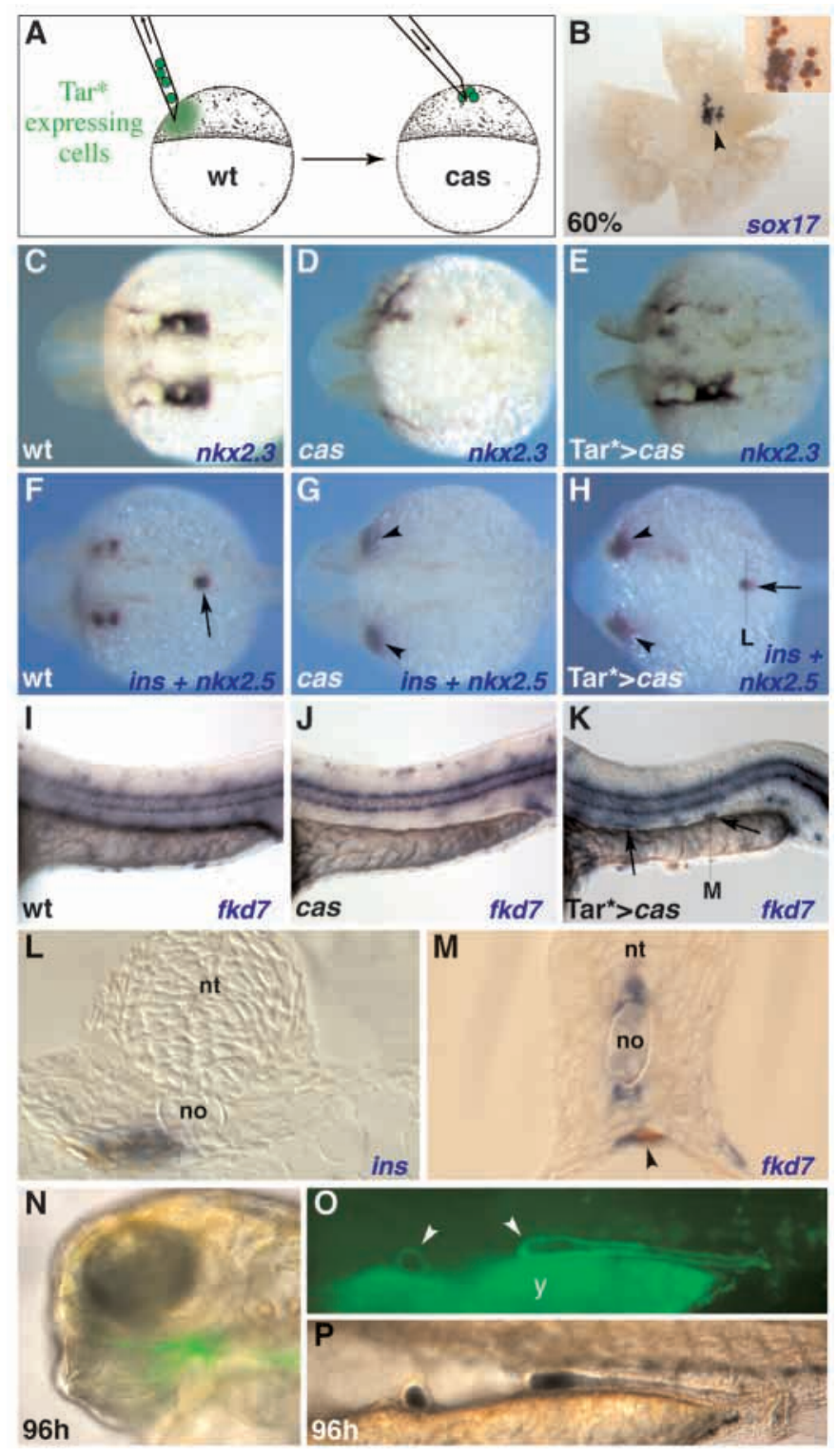

had reached positions corresponding to that of endodermal derivatives. Furthermore, region-specific endodermal markers were restored in register with their AP position. For instance, the pharyngeal region expressed $n k x 2.3$ (Fig. 7C-E) and $f k d 7$ (not shown), the midgut region expressed the insulin gene (Fig. $7 \mathrm{~F}-\mathrm{H}$ ) and the hindgut region expressed $f k d 7$ (Fig. 7I-K). Expression of these markers was found within grafted cells (Fig. 7L,M and data not shown). At 96 hpf, activated wild-type cells (green) could restore portions of endodermal derivatives, such as the pharyngeal epithelium (Fig. 7N) or segments of intestine (Fig. 7O,P). Grafting control, non-activated cells, expressing only GFP, also led to rescue, but with a lower efficiency since only a fraction of grafted cells, in a portion of the embryos ( $38 \%, n=8$ embryos) contributed to endoderm (data not shown). These results demonstrate, firstly, that since cas mutants are totally lacking endoderm, the endoderm of the host is not absolutely required for activated cells to differentiate into endoderm, secondly, that wild-type cells, particularly when activated, can accomplish the full endodermal program within a cas-deficient environment. We conclude that, once the Nodal/Tar pathway is activated, cas is not absolutely required outside the endodermal progenitors to allow their later differentiation.

\section{Endoderm formation can restore cardiac fusion in casanova mutants}

Compared to controls (Fig. 8A,D), cas mutants display a cardia bifida, resulting from the absence of fusion of the hemicardia, which stay lateral instead of migrating to the midline (Fig. 8B,E) (Chen et al., 1996). Results established in chick (Linask and Lash, 1988), as well as our own analysis of the cardia bifida present in оер zebrafish embryos (Peyrieras et al., 1998) suggest that this migration defect in cas mutants could be secondary to the absence of endoderm. Alternatively, cas activity could be required within non endodermal territories to facilitate the fusion process. The capacity to restore at high

Fig. 7. Wild-type activated cells can form endoderm in cas mutants. (A) At the blastula stage, a few wild-type cells expressing Tar* were transplanted to a cas host. (B) Flat-mount of a young gastrula $(60 \%$ epiboly) showing the expression of sox 17 (blue) by activated cells (brown nuclear staining). Notice the absence of endogenous sox17 expression in cas embryos. The inset shows a close-up view of the region containing the grafted cells. (C-K) Expression of $n k x 2.3$, insulin and $f k d 7$ in $24 \mathrm{hpf}$ wild-type (C,F,I), cas (D,G,J) and grafted cas $(\mathrm{E}, \mathrm{H}, \mathrm{K})$ embryos. (C-E) The pharyngeal expression of $n k x 2.3$ (C) is absent in cas embryos (D). This expression can be restored by the graft of wild-type activated cells (E). (F-H) Pancreatic expression of insulin (arrow in F), which is absent in cas embryos (G), can be restored by the graft (arrow in $\mathrm{H}$ ). The expression of $n k x 2.5$ (arrowheads) identifies the cardia bifida of cas embryos and was used to genotype them. (I-K) The expression of $f k d 7$ in the gut (I) is lost in cas embryos (J). This expression can be restored by wild-type activated cells (arrows in K). (L,M) Transverse sections through the pancreatic and gut regions of grafted cas embryos showing that the expression of the insulin and $f k d 7$ (blue, arrowhead in $\mathrm{M}$ ) is restricted to grafted cells (brown nuclear staining). (N-P) Live grafted cas embryos. At 96 hpf, grafted cells (green) differentiate into endodermal derivatives such as pharyngeal epithelium or segments of gut (arrowheads). In O, the yolk (y) appears green because of its autofluorescence. $(\mathrm{C}-\mathrm{H})$ Dorsal views, anterior to the left. (I-K,N-P) Lateral views, anterior to the left, dorsal to the top. no, notochord; nt, nerve tube. 
Fig. 8. Restoring wild-type endoderm in cas mutants allows heart fusion. (A-C) $n k x 2.5$ and $f k d 7$ expression were detected by in situ hybridisation in 24 hpf embryos. (A) Wild-type embryo, (B) control cas embryo, (C) and cas embryo grafted with wild-type activated cells. Arrowheads indicate the heart in wild-type embryos, hemicardia in cas and fused hemicardia in grafted cas embryos.

(D-F) Transverse sections through the heart region of $24 \mathrm{hpf}$ embryos stained for $n k x 2.5$ expression. (D) Wild-type embryo with a single heart. (E) Control cas embryo with two hemicardia in lateral position. (F) cas embryo grafted with wild-type activated cells. The two hemicardia have fused at the midline. The inset
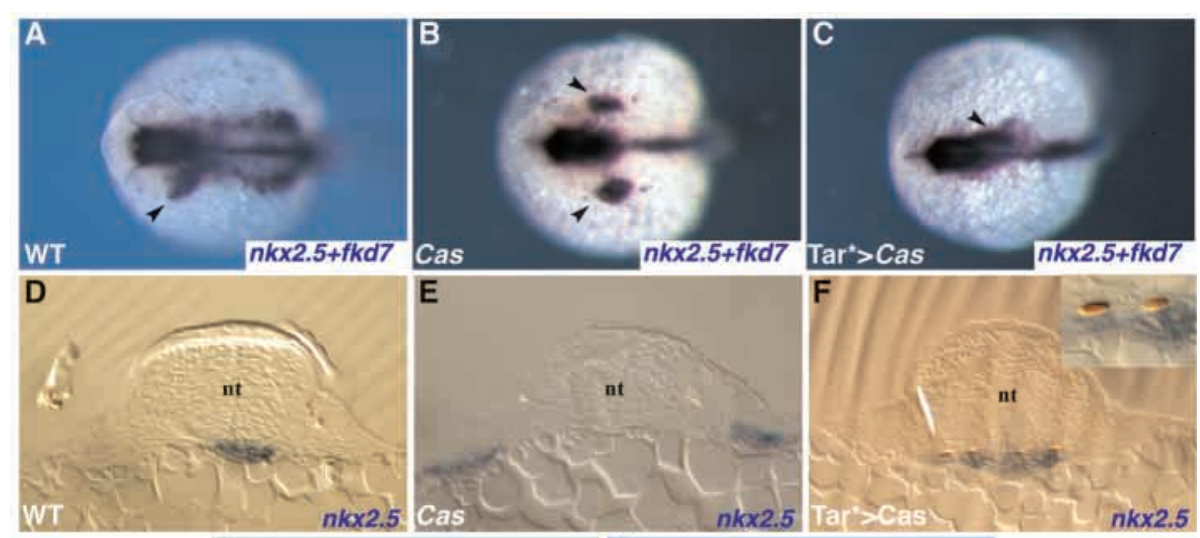

is a close-up view of the heart region showing that grafted cells (brown nuclei) are not incorporated into the heart but are endodermal derivatives overlaying it. (G-H) cas embryos grafted with wild-type activated cells exhibiting an axis duplication. Arrowheads point to the unfused hemicardia in the primary axis $(\mathrm{H})$ and the arrow indicates the heart in the secondary axis $(\mathrm{G}$, H). (G) Lateral view of a live embryo, anterior left. (H) Ventral view, following staining for $n k x 2.5$ expression. nt, nerve tube.

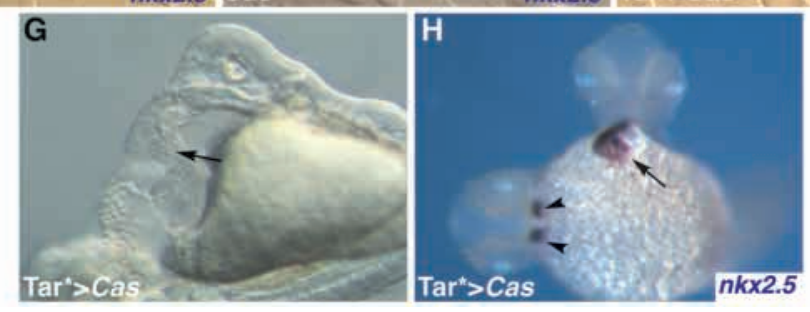

efficiency endoderm tissues in cas embryos by grafting wildtype activated cells offered us the opportunity to test this hypothesis. We analysed the behaviour of the heart precursors in cas embryos in which control or Tar*-activated wild-type cells had been grafted. At $24 \mathrm{hpf}$, myocardial cells expressed the marker $n k x 2.5$ (Lee et al., 1996). In cas unmanipulated embryos $(100 \%, n=150)$ or in cas embryos grafted with control cells $(100 \%, n=26), n k x 2.5$-positive cells remained on both sides of the embryos, reflecting the position of the unfused hemicardia (Fig. 8B,E). By contrast, in $48 \%(n=23)$ of cas embryos grafted with Tar*-activated wild-type cells, the hemicardia had moved closer or even fused at the midline (Fig. $8 \mathrm{C}, \mathrm{F})$. Transverse sections through the heart region showed that grafted cells were located in the endoderm and did not contribute to heart tissues (Fig. 8F). The graft of activated cells led, in a fraction of the embryos $(n=5)$ to the formation of a secondary axis, by induction of a second organizer (Peyrieras et al., 1996). The primary axis harboured a cardia bifida as expected. Quite strikingly, the secondary axis exhibited a single, beating heart including two chambers and an endothelium (Fig. 8G,H). In one of these embryos, blood circulation was observed in the head region of the secondary axis, demonstrating that the heart was functional. We conclude from these experiments that cas activity is not absolutely required within heart precursors cells to allow proper hemicardia fusion. Rather, since the graft of endoderm mediated by the implantation of wild-type activated cells can restore a functional heart, it strongly suggests that the cardia bifida observed in cas mutants is due to the absence of endoderm.

\section{DISCUSSION}

\section{Activation of Nodal/Tar signalling and commitment to an endodermal fate}

We show here that, consistent with the fact that commitment to a particular germ layer occurs during gastrulation in vertebrates, endoderm commitment occurs at the onset of gastrulation. However, endoderm commitment appears to occur before mesoderm commitment and may thus represent one of the earliest determination events affecting the zebrafish embryo (Ho and Kimmel, 1993). Endoderm formation, at the margin of the embryo, relies on the activity of the Nodal pathway which is both necessary and sufficient to induce endoderm. In particular, activation of this pathway by injection of low levels of the activated form of the TGF $\beta$ type I receptor Tar* drives blastomeres to an endodermal fate. In this process, Nodal signalling could only be important for the initial specification of endoderm progenitors at the margin of the embryo. Other factors, released from the margin, could control the maintenance of endoderm. Alternatively, Nodal signalling could also be important for maintaining the endodermal identity during gastrulation and thus control commitment to endoderm. By grafting a small group of activated cells into different locations of host embryos, we obtained direct evidence that upon activation of Nodal signalling, cells engage in the process of determination to an endodermal fate. Transplantations were performed by the late blastula stage, indicating that, from this time on, activated cells are already engaged in the process leading to determination. Whether this reflects a state of commitment from the start of activation of the Nodal pathway, or action at a later stage, cannot be ascertained at this point. In vivo, both the Nodal-related ligands Cyc and Sqt, as well as the receptor Tar are expressed in endoderm precursors before they translocate to the hypoblast region, but their expression is markedly downregulated after marginal cells have involuted. We thus suspect that these elements of Nodal signalling act primarily between their onset of expression at the midblastula transition and the onset of gastrulation.

Nodal/Tar signals may modify directly the fate of the activated cells in either an autonomous or non-autonomous fashion. The graft of a group of activated cells or activated 
single cells demonstrates unambiguously that the process is indeed autonomous: activated cells express endoderm markers when grafted to an ectopic site and further differentiate into endodermal derivatives. In addition, activated cells either isolated or in groups cannot induce endodermal markers in neighbouring cells. The requirements for the establishment of this endodermal identity was examined in detail. The requirement for the YSL in the identity of Nodal/Tar-activated cells was first investigated, since this syncitium has an endoderm-inducing activity, expresses Nodal-related genes transiently and is the major substratum for the migration of endoderm cells. We have shown, by grafting endodermcommitted blastomeres or Tar*-expressing cells into the animal region of host embryos and dissecting the animal caps, that the YSL is not required to maintain the endodermal identity of these cells during gastrulation. Consistent with this result, whole blastoderms separated from the YSL can maintain the expression of endodermal markers (T. Aoki and F. Rosa, unpublished data). Thus the YSL may release, at an early stage, endoderm inducing factors, likely related to the Nodals, but does not appear necessary during gastrulation for maintaining endoderm identity. Likewise, the requirement for endogenous endoderm was also analysed by grafting activated wild-type cells into cas embryos, which are devoid of endoderm. Activated cells expressed endoderm markers at gastrulation and differentiated into endoderm at later stages. We have carried out similar experiments using oep mutant hosts, which are also deficient in endoderm, and obtained similar results (J. Mathieu and F. M. R., unpublished). Thus endogenous endoderm is not required for Nodal/Tar-activated cells to keep their identity and to differentiate into endoderm. This is in contrast to recent experiments carried out in Xenopus (Clements and Woodland, 2000), which show that sox17expressing cells adopt an endodermal identity, even when originating from an ectodermal region, but later die if they cannot join the resident endoderm population. This difference in late behaviour may either stem from a developmental difference between Xenopus and zebrafish or may suggest that the activation of the entire Nodal/Tar pathway provides necessary cues, which are not provided by the activation of the downstream pathway mediated by Sox 17. Whatever the origin of this difference, our results clearly demonstrate that the induction of endoderm by activation of the Nodal/Tar pathway is largely autonomous.

\section{Activation of Nodal signalling triggers the specific translocation of blastomeres to the surface of the YSL}

Endoderm progenitors form at the margin of the embryo under the influence of Nodal-related signals. Very soon after their fate can be established, endoderm progenitors sink into the depth of the blastoderm to reach the surface of the YSL, on which they actively migrate during gastrulation. It was not clear whether endoderm cells need to be close to the margin to achieve these translocation movements. It is not established either whether the marginal YSL has specific properties as compared to the animal pole YSL, which allow the proper recognition by endoderm cells. By transplanting endodermfated activated cells or ECB to the animal pole of zebrafish blastulae, we have observed that, soon after the onset of gastrulation, these transplanted cells reach the YSL.
Furthermore, they do not require involution through the margin of the embryo to reach the YSL. These results, which were also observed when grafting at any position of the host embryo, clearly demonstrate first that the animal pole and marginal YSL represent equal targets for the translocation of endoderm cells, although we cannot exclude that endoderm cells translocate faster at the margin than at the animal pole. Second, they show that once activated, cells can directly reach their target site, without the need for specific cues provided by the margin of the embryo. Nodal-related signals have also been reported to control the segregation between mesoderm and ectoderm, thus Nodal signals may play a general role in controlling cell position in the gastrula (Wacker et al., 2000).

Analysis of the behaviour of activated cells clearly raises two issues. First we need to understand how they can sort from their neighbours and second how they direct their movement to the YSL. One possibility is that activated cells express a range of adhesive/repulsive molecules that identify them as endodermal. In this case, they would be progressively expelled from the animal pole to become interspersed within the endodermal cells. This sorting-out process (Townes and Holtfreter, 1955) had been proposed by Ho to explain the exclusion of hypoblastic cells from the epiblast (Ho, 1993) and was more recently suggested to explain the behaviour of Xsox17-expressing blastomeres in Xenopus (Clements and Woodland, 2000). Such a sorting-out process, if involved, does not rely on the presence of endoderm, since activated cells translocate to the YSL in a cas environment. Alternatively, specific translocation to the YSL may result from a high mobility of activated cells or from guidance cues provided by the environment and/or the YSL. The delineation of this process is currently under investigation in our laboratory. In any case, the properties of activated cells, combined with the use of mutants, should greatly facilitate a better understanding of the cellular and molecular processes controlling cell movements during gastrulation, a major open question.

\section{Activated cells give rise to correctly patterned endodermal derivatives}

In 24 hpf embryos, activated cells grafted to the margin of the blastoderm are found in AP positions consistent with the fate map established at the beginning of gastrulation, indicating that, during gastrulation, they behave like endogenous endoderm. Furthermore, when transplanted into either wildtype or cas recipient embryos, they correctly express regionalized endodermal markers such as $n k x 2.3, f k d 7$ or insulin. In particular, no marked ectopic expression of these genes has been observed. Thus, endodermal tissues derived from activated cells seem to be correctly patterned along the anteroposterior axis. This AP pattern may be established in different non-exclusive ways. One possibility is that endoderm obtains its regional identity via an autonomous program, without regard to adjacent germ layers. For instance, subsets of endoderm cells could be predetermined, at the onset of gastrulation, to migrate up to the animal pole or to remain next to the margin and to differentiate accordingly. Predetermination appears highly unlikely since activated cells, although they can give rise to posterior endodermal derivatives when grafted to the margin, do not when grafted to the animal pole. A second possibility is that the AP identity of endodermal cells could be defined as a consequence of the length or 
duration of migration during gastrulation. This can also be ruled out because cells grafted to the animal pole do not have to undergo extensive migration and still differentiate according to their AP position. The third, most likely, possibility is that cell-to-cell interactions provide positional information for the endoderm. One good example in this respect is the regulation, in the prospective anterior endoderm, of the gene her5, which involves both positive and negative extracellular signals (BallyCuif et al., 2000). One possibility is that endoderm is submitted to self-patterning by the release of planar signals emanating from a specific region of the endoderm germ layer. Here we show that the small fragments of endoderm restored in cas mutants, otherwise deficient of endoderm, are normally patterned. It thus seems unlikely that they acquired their AP identity through patterning signals solely emitted by the endoderm. Rather, patterning signals are most likely provided by the environment of endoderm cells, irrespective of the germ layer considered. In agreement with this idea, recent results established in Xenopus have shown that endoderm retains its AP identity when grafted along with its mesodermal and ectodermal sheath, but does not when grafted alone (Zeynali et al., 2000). Likewise, in mice, in vitro explant assays have shown that adjacent germ layers provide diffusible signals that induce organ-specific gene expression in endoderm. In particular, FGF4 could act as a morphogen for posterior endoderm since it induces different markers in a dosedependent manner (Wells and Melton, 2000). These in vitro approaches, as well as our own studies on the regulation of the her 5 gene have opened the way to a better understanding of endoderm patterning. In this context, the use of Nodal/Taractivated cells should prove a very useful tool in understanding the mechanisms controlling this crucial patterning process.

\section{A role for endoderm in heart morphogenesis}

Endoderm is thought to play an essential role in the control of organogenesis. Our work has provided the opportunity to examine the specific function of endoderm in the organogenesis of the heart. In addition to the absence of endoderm, cas embryos exhibit an abnormal morphogenesis of the heart, leading to cardia bifida. By introducing wild-type endoderm into cas embryos, normal heart morphogenesis can be rescued, providing direct evidence that the cardia bifida is not due to the absence of cas activity in heart precursors, but more likely in surrounding tissues. In addition, both the lack of endoderm in cas embryos and the restoration of heart morphogenesis by introduction of wild-type endoderm strongly support a role for endoderm itself in mediating cardiac fusion. This interpretation is consistent with previous reports, including ours. Quails with abnormal endodermal apoptosis display a severe cardiac phenotype sometimes leading to cardia bifida. This phenotype can be rescued by grafting wild-type anterior endoderm (Ghatpande et al., 2000). Several mutants, including Gata4 in the mouse and fau, bon and oep in zebrafish, exhibiting cardia bifida, have an associated defective endodermal tissue. Chimera analysis have shown that expression of gata4 restricted to endoderm is sufficient to rescue heart formation (Narita et al., 1997). In studying the cardia bifida exhibited by zebrafish oep mutants, we have shown that the rescue of endoderm tissue in these mutants results in the non-autonomous fusion of the hemicardia
(Peyrieras et al., 1998). Taken together these results support a major role for endoderm in heart hemicardia fusion. Although this role could be indirect, the immediate proximity of pharyngeal endoderm and the heart precursors suggests that close interactions between the two tissues allow proper migration of the hemicardia to the midline and subsequent fusion to generate a functional heart. One possibility is that endoderm provides a simple substratum for migration of hemicardia to the midline under the influence of non endodermal cues. Another possibility is that endoderm provides both a substratum and directional cues. A third possibility is that heart hemicardia undergo passive migration toward the midline, carried by the extensive migration of pharyngeal endoderm below the head (Peyrieras et al., 1998), a phenomenon we also observe when grafting Tar*-activated cells. We clearly cannot choose between these possibilities but we notice that, in weak alleles of $f a u$, pharyngeal endoderm is present but exhibits defective convergence, a phenomenon supporting the third possibility. In conclusion, our results clearly show that grafts of wild-type activated cells into mutants devoid of endoderm is a powerful tool for understanding the organising and/or inducing activities of endoderm during development.

We thank F. Argenton, J. Campos-Ortega, U. Straehle, D. Stainier, A. Schier for plasmids and fish strains. We are indebted to L. BallyCuif, M. Wassef and P. Mourrain for a critical reading of this manuscript and to H. Sawyer for participating in the very early phase of this work. We are grateful to F. Chelgoum and F. Bouallague for care of the fish. This work was supported by a grant from ARC.

\section{REFERENCES}

Alexander, J., Rothenberg, M., Henry, G. L. and Stainier, D. Y. (1999). casanova plays an early and essential role in endoderm formation in zebrafish. Dev. Biol. 215, 343-357.

Alexander, J. and Stainier, D. Y. (1999). A molecular pathway leading to endoderm formation in zebrafish. Curr. Biol. 9, 1147-1157.

Argenton, F., Zecchin, E. and Bortolussi, M. (1999). Early appearance of pancreatic hormone-expressing cells in the zebrafish embryo. Mech. Dev. 87, 217-221.

Bally-Cuif, L., Goutel, C., Wassef, M., Wurst, W. and Rosa, F. (2000). Coregulation of anterior and posterior mesendodermal development by a hairy-related transcriptional repressor. Genes Dev. 14, 1664-1677.

Chen, J. N., Haffter, P., Odenthal, J., Vogelsang, E., Brand, M., van Eeden, F. J., Furutani-Seiki, M., Granato, M., Hammerschmidt, M., Heisenberg, C. P. et al. (1996). Mutations affecting the cardiovascular system and other internal organs in zebrafish. Development 123, 293-302.

Clements, D. and Woodland, H. R. (2000). Changes in embryonic cell fate produced by expression of an endodermal transcription factor, Xsox 17 . Mech. Dev. 99, 65-70.

Dale, L. (1999). Vertebrate development: Multiple phases to endoderm formation. Curr. Biol. 9, R812-815.

Dickmeis, T., Mourrain, P., Saint-Etienne, L., Fischer, N., Aanstad, P., Clark, M., Strahle, U. and Rosa, F. (2001). A crucial component of the endoderm formation pathway, CASANOVA, is encoded by a novel soxrelated gene. Genes Dev. 15, 1487-1492.

Feldman, B., Gates, M. A., Egan, E. S., Dougan, S. T., Rennebeck, G., Sirotkin, H. I., Schier, A. F. and Talbot, W. S. (1998). Zebrafish organizer development and germ-layer formation require nodal-related signals [see comments]. Nature 395, 181-185.

Ghatpande, S., Ghatpande, A., Zile, M. and Evans, T. (2000). Anterior endoderm is sufficient to rescue foregut apoptosis and heart tube morphogenesis in an embryo lacking retinoic acid. Dev. Biol. 219, 59-70.

Grapin-Botton, A. and Melton, D. A. (2000). Endoderm development: from patterning to organogenesis. Trends Genet. 16, 124-130. 
Gritsman, K., Talbot, W. S. and Schier, A. F. (2000). Nodal signaling patterns the organizer. Development 127, 921-932.

Gurdon, J. B. (1988). A community effect in animal development. Nature 336, 772-774.

Hauptmann, G. and Gerster, T. (1994). Two-color whole-mount in situ hybridization to vertebrate and Drosophila embryos. Trends Genet. 10, 266

Heasman, J., Wylie, C. C., Hausen, P. and Smith, J. C. (1984). Fates and states of determination of single vegetal pole blastomeres of X. laevis. Cell 37, 185-194.

Ho, R. K. and Kimmel, C. B. (1993). Commitment of cell fate in the early zebrafish embryo. Science 261, 109-111.

Joubin, K. and Stern, C. D. (1999). Molecular interactions continuously define the organizer during the cell movements of gastrulation. Cell 98, 559571

Kikuchi, Y., Agathon, A., Alexander, J., Thisse, C., Waldron, S., Yelon, D., Thisse, B. and Stainier, D. Y. (2001). casanova encodes a novel Sox-related protein necessary and sufficient for early endoderm formation in zebrafish. Genes Dev. 15, 1493-1505.

Kikuchi, Y., Trinh, L. A., Reiter, J. F., Alexander, J., Yelon, D. and Stainier, D. Y. (2000). The zebrafish bonnie and clyde gene encodes a Mix family homeodomain protein that regulates the generation of endodermal precursors. Genes Dev. 14, 1279-1289.

Kimelman, D. and Maas, A. (1992). Induction of dorsal and ventral mesoderm by ectopically expressed Xenopus basic fibroblast growth factor. Development 114, 261-269.

Kimmel, C. B., Ballard, W. W., Kimmel, S. R., Ullmann, B. and Schilling, T. F. (1995). Stages of embryonic development of the zebrafish. Dev. Dyn. 203, 253-310.

Kimmel, C. B., Warga, R. M. and Schilling, T. F. (1990). Origin and organization of the zebrafish fate map. Development 108, 581-594.

Lee, K. H., Xu, Q. and Breitbart, R. E. (1996). A new tinman-related gene, nkx2.7, anticipates the expression of nkx2.5 and nkx2.3 in zebrafish heart and pharyngeal endoderm. Dev. Biol. 180, 722-731.

Linask, K. K. and Lash, J. W. (1988). A role for fibronectin in the migration of avian precardiac cells. II. Rotation of the heart-forming region during different stages and its effects. Dev. Biol. 129, 324-329.

Muller, F., Albert, S., Blader, P., Fischer, N., Hallonet, M. and Strahle, U. (2000). Direct action of the nodal-related signal cyclops in induction of sonic hedgehog in the ventral midline of the CNS. Development 127, 38893897.

Muller, M., Weizsacker, E. and Campos-Ortega, J. A. (1996). Transcription of a zebrafish gene of the hairy-Enhancer of split falimy delineates the midbrain anlage in the neural plate. Dev. Genes Evol. 206, 153-160.

Narita, N., Bielinska, M. and Wilson, D. B. (1997). Wild-type endoderm abrogates the ventral developmental defects associated with GATA-4 deficiency in the mouse. Dev. Biol. 189, 270-274.

Odenthal, J. and Nusslein-Volhard, C. (1998). fork head domain genes in zebrafish. Dev. Genes Evol. 208, 245-258.
Peyrieras, N., Lu, Y., Renucci, A., Lemarchandel, V. and Rosa, F. (1996). Inhibitory interactions controlling organizer activity in fish. $C R$ Acad. Sci. Iii 319, 1107-1112.

Peyrieras, N., Strahle, U. and Rosa, F. (1998). Conversion of zebrafish blastomeres to an endodermal fate by TGF-beta-related signaling. Curr Biol. 8, 783-786.

Rebagliati, M. R., Toyama, R., Haffter, P. and Dawid, I. B. (1998). cyclops encodes a nodal-related factor involved in midline signaling. Proc. Natl. Acad. Sci. USA 95, 9932-9937.

Reiter, J. F., Kikuchi, Y. and Stainier, D. Y. (2001). Multiple roles for Gata5 in zebrafish endoderm formation. Development 128, 125-135.

Renucci, A., Lemarchandel, V. and Rosa, F. (1996). An activated form of type I serine/threonine kinase receptor TARAM-A reveals a specific signalling pathway involved in fish head organiser formation. Development 122, 3735-3743.

Rodaway, A., Takeda, H., Koshida, S., Broadbent, J., Price, B., Smith, J. C., Patient, R. and Holder, N. (1999). Induction of the mesendoderm in the zebrafish germ ring by yolk cell-derived TGF-beta family signals and discrimination of mesoderm and endoderm by FGF. Development $\mathbf{1 2 6}$, 3067-3078.

Serbedzija, G. N., Chen, J. N. and Fishman, M. C. (1998). Regulation in the heart field of zebrafish. Development 125, 1095-1101.

Spemann, H. (1938). Embryonic Development and Induction. Reprinted in 1967, New York: Hafner.

Strahle, U., Blader, P., Henrique, D. and Ingham, P. W. (1993). Axial, a zebrafish gene expressed along the developing body axis, shows altered expression in cyclops mutant embryos. Genes Dev. 7, 1436-1446.

Townes, P. L. and Holtfreter, J. (1955). Directed movements and selective adhesion of embryonic amphibian cells. J. Exp. Zool. 128, 53-120.

Wacker, S., Grimm, K., Joos, T. and Winklbauer, R. (2000). Development and control of tissue separation at gastrulation in Xenopus. Dev. Biol. 224 428-439.

Warga, R. M. and Nusslein-Volhard, C. (1999). Origin and development of the zebrafish endoderm. Development 126, 827-838.

Wells, J. M. and Melton, D. A. (1999). Vertebrate endoderm development. Annu. Rev. Cell Dev. Biol. 15, 393-410.

Wells, J. M. and Melton, D. A. (2000). Early mouse endoderm is patterned by soluble factors from adjacent germ layers. Development 127, 15631572.

Westerfield, M. (1995). The Zebrafish Book. Univ of Oregon Press, Eugene.

Wylie, C. C., Snape, A., Heasman, J. and Smith, J. C. (1987). Vegetal pole cells and commitment to form endoderm in Xenopus laevis. Dev. Biol. 119 , 496-502.

Yasuo, H. and Lemaire, P. (1999). A two-step model for the fate determination of presumptive endodermal blastomeres in Xenopus embryos. Curr. Biol. 9, 869-879.

Zeynali, B., Kalionis, B. and Dixon, K. E. (2000). Determination of anterior endoderm in Xenopus embryos. Dev. Dyn. 218, 531-536. 